\title{
Histone deacetylase inhibitor suberoyl bis-hydroxamic acid suppresses cell proliferation and induces apoptosis in breast cancer cells
}

\author{
XINMIAO YANG ${ }^{1}$, NING ZHANG ${ }^{1}$, ZELIANG SHI ${ }^{2}$, ZHANGYU YANG ${ }^{2}$ and XICHUN HU ${ }^{3}$ \\ ${ }^{1}$ Department of Medical Oncology, Minhang Branch of Fudan University Shanghai Cancer Center, Shanghai 200240; \\ ${ }^{2}$ Department of Radiation Oncology, Affiliated Sixth People's Hospital, Shanghai Jiao Tong University, Shanghai 200233; \\ ${ }^{3}$ Department of Medical Oncology, Fudan University Cancer Hospital, Shanghai 200032, P.R. China
}

Received February 21, 2014; Accepted November 14, 2014

DOI: $10.3892 / \mathrm{mmr} .2014 .3076$

\begin{abstract}
Suberoyl bis-hydroxamic acid (SBHA) is a histone deacetylase inhibitor that has shown anticancer activity against numerous types of human cancer. The aim of the current study was to explore the effects of SBHA on the proliferation and apoptosis of breast cancer cells. MCF-7 breast cancer cells were treated with different concentrations of SBHA and tested for cell viability, apoptosis and gene expression changes. The results showed that SBHA significantly inhibited the proliferation of MCF-7 cells in a concentration-dependent manner, as determined using a Cell Counting kit-8 assay. SBHA-treated MCF-7 cells showed $\mathrm{G}_{0} / \mathrm{G}_{1}$ cell-cycle arrest, coupled with elevated expression levels of p21 and p27 proteins. Hoechst 33258 staining revealed cell shrinkage, chromosomal condensation and nuclear fragmentation in MCF-7 cells treated with SBHA. Flow cytometric analysis of Annexin V-stained cells showed that SBHA treatment induced apoptotic cell death in a concentration-dependent manner. Western blot analysis confirmed the upregulation of Bax and the downregulation of Bcl-2 by SBHA. In conclusion, these results indicate that SBHA exerts cytotoxic effects against human breast cancer cells, which involves the modulation of p21, p27 and Bcl-2 family proteins, consequently leading to cell-cycle arrest and apoptosis.
\end{abstract}

\section{Introduction}

Breast cancer is one of the most common types of malignant disease affecting females worldwide, causing $>450,000$

Correspondence to: Dr Xinmiao Yang, Department of Medical Oncology, Minhang Branch of Fudan University Shanghai Cancer Center, 106 Ruili Road, Shanghai 200240, P.R. China

E-mail: dr.editor.bio@gmail.com

Key words: anticancer therapy, Bcl-2 family, cell cycle arrest, histone deacetylase mortalities each year (1). The current treatment modalities for breast cancer include surgical resection, adjuvant radiotherapy and advanced chemotherapeutic agents, including cisplatin, pacliataxel, carboplatin, bevacizumab, doxorubicin, cyclophosphamide, docetaxel and epirubicin (2). Despite advances in treatment strategies, mortality from breast cancer remains high. Therefore, there is a clear requirement for the development of novel therapeutic agents.

Histone acetyltransferases (HATs) and histone deacetylases (HDACs) are known to have opposing roles in the regulation of global gene expression via an epigenetic mechanism (3). HATs catalyze the acetylation of lysine residues in histone tails, facilitating and sustaining gene transcription, while HDACs are responsible for the removal of acetyl groups from the $\varepsilon$-amine of lysine residues of histone tails, culminating in prevention of gene transcription. HDAC inhibitors that have the ability to block the activities of HADCs have emerged as effective anticancer agents (4). Their anticancer effects are associated with the modulation of various cell behaviors, including differentiation, cell cycle progression, apoptosis and angiogenesis $(5,6)$. At the molecular level, HDAC inhibitors can affect the expression of a large number of genes, particularly key regulators of apoptosis and the cell cycle such as p21, p27, Bax, and Bcl-2 (5-7).

Suberoylanilide hydroxamic acid (SAHA) and trichostatin A (TSA) are two of the most investigated HDAC inhibitors and have shown cytotoxic effects against a number of tumor types, including breast cancer $(8,9)$. Suberoyl bis-hydroxamic acid (SBHA) has a similar structure to SAHA and TSA, and has been found to prevent tumor growth in several types of malignancies, such as medullary thyroid cancer (10) and lung cancer (11). You and Park (11) reported that SBHA is capable of inhibiting the growth of A549 lung cancer cells via caspase-dependent apoptosis. In addition, in MCF-7 breast cancer cells, SBHA treatment causes a significant level of apoptosis (12). However, the antitumor activity of SBHA in breast cancer cells and its associated molecular mechanisms are not completely understood. In the current study, the cytotoxic effect of SBHA against MCF-7 breast cancer cells was evaluated and its impact on cell cycle progression and apoptosis was examined. 


\section{Materials and methods}

Cells and reagents. MCF-7 breast cancer cells were purchased from the American Type Culture Collection (Manassas, VA, USA). Fetal bovine serum (FBS), RPMI-1640 medium, penicillin, streptomycin, Hoechst 33258, propidium iodide (PI) and dimethyl sulfoxide(DMSO) were obtained from Sigma-Aldrich (St. Louis, MO, USA); SBHA was purchased from Calbiochem (San Diego, CA, USA); Cell Counting kit-8 (CCK-8) was obtained from Dojindo Molecular Technologies (Kumamoto, Japan); an Annexin-FITC kit was purchased from Beckman Coulter (Fullerton, CA, USA) and DNase-free RNase was from Roche Applied Science (Penzburg, Germany). Mouse monoclonal antibodies against p21 (sc-271532), p27 (sc-1641), Bcl-2 (sc-7382), Bax (sc-20067) and $\beta$-actin (sc-130301) were purchased from Santa Cruz Biotechnology, Inc. (Santa Cruz, CA, USA). Horseradish peroxidase-conjugated goat anti-mouse IgG antibody was obtained from Rockland (Gilbertsville, PA, USA).

Cell culture and treatment. MCF-7 cells were cultured in RPMI-1640 medium supplemented with $10 \%$ heat-inactivated FBS, penicillin $(100 \mathrm{U} / \mathrm{ml})$ and streptomycin $(100 \mu \mathrm{g} / \mathrm{ml})$. Cells were maintained in a humidified incubator with $5 \% \mathrm{CO}_{2}$ and $95 \%$ air at $37^{\circ} \mathrm{C}$ and subcultured every 3-4 days. Twenty-four hours after plating, cells were treated with different concentrations of SBHA for $72 \mathrm{~h}$ and subjected to cell proliferation, apoptosis and gene expression analysis. Untreated cells were used as the control.

CCK- 8 assay. The effect of SBHA on cell proliferation was determined with the CCK-8 cell proliferation assay kit. Briefly, MCF-7 cells were seeded in 96-well plates at a density of $5 \times 10^{3}$ cells/well and incubated for $24 \mathrm{~h}$. Following incubation, the cells were exposed to $10,40,60,80$, or $100 \mu \mathrm{M}$ SBHA for $72 \mathrm{~h}$. Untreated cells served as the control. Cells were incubated for a further $2 \mathrm{~h}$ in the presence of CCK- 8 reagent. The absorbance (optical density) was measured at a wavelength of $450 \mathrm{~nm}$ using a microplate reader (Bio-Rad 3550; Bio-Rad Laboratories, Inc., Hercules, CA, USA).

Cell cycle analysis. Following treatment, cells were trypsinized, washed and fixed with $75 \%$ ice-cold ethanol. Cells were pelleted by centrifugation at $300 \mathrm{x}$ g for $5 \mathrm{~min}$ and suspended in DNA staining solution containing $20 \mu \mathrm{g} / \mathrm{ml} \mathrm{PI}$ and $20 \mu \mathrm{g} / \mathrm{ml}$ DNase-free RNase. After incubation at $37^{\circ} \mathrm{C}$ for $15-30 \mathrm{~min}$, cells were analyzed on a FACSCalibur flow cytometer (BD Biosciences, San Jose, CA, USA). Cell cycle distribution was determined and calculated using CellQuest 5.2 software (BD Biosciences).

Characterization of apoptotic morphology by Hoechst 33258 staining. Cells were grown on sterile cover slips in 6-well tissue culture plates. When the cells reached $60-80 \%$ confluence, they were treated with SBHA for $72 \mathrm{~h}$. After washing with phosphate-buffered saline Invitrogen (Carlsbad, CA, USA), cells were fixed with $4 \%$ paraformaldehyde for $10 \mathrm{~min}$, washed, and stained with Hoechst $33258(5 \mu \mathrm{g} / \mathrm{ml})$ for $5 \mathrm{~min}$ at room temperature. Cells were mounted prior to examination using a DMIRE2 fluorescence microscope (Leica, Bensheim, Germany).
Apoptosis analysis by Annexin V/PI staining. Cells were seeded onto 6-well plates and exposed to different concentrations of SBHA ranging from $20-120 \mu \mathrm{M}$ for $72 \mathrm{~h}$. Cells were harvested through trypsinization, washed and centrifuged at $300 \mathrm{x}$ g for $5 \mathrm{~min}$. The cell pellet was resuspended in $1 \mathrm{X}$ binding buffer. The cell sample solution $(100 \mu \mathrm{l})$ was incubated with $1 \mu \mathrm{l}$ fluorescein isothiocyanate (FITC)-conjugated Annexin V and $5 \mu \mathrm{PI}$ for $15 \mathrm{~min}$ at $4^{\circ} \mathrm{C}$ in the dark. The $1 \mathrm{X}$ binding buffer (400 $\mu \mathrm{l}$ ) was added to each sample tube and the samples were analyzed on a FACSCalibur flow cytometer using CellQuest software.

Western blot analysis. After treatment, cells were lysed in lysis buffer $(10 \mathrm{mmol} / 1$ Tris, $\mathrm{pH} \mathrm{7.4;} 130 \mathrm{mmol} / 1 \mathrm{NaCl}, 1 \%$ Triton $\mathrm{X}$, $10 \mathrm{mmol} / \mathrm{l} \mathrm{NaF}, 10 \mathrm{mmol} / \mathrm{l} \mathrm{NaPi}, 10 \mathrm{mmol} / \mathrm{l} \mathrm{NaPPi}$, and $1.5 \mathrm{mmol} / \mathrm{l}$ EDTA) supplemented with the protease inhibitor $\operatorname{aprotinin}(2 \mathrm{mg} / \mathrm{l})$ and phosphatase inhibitors leupeptin $(5 \mathrm{mg} / \mathrm{l})$ and phenylmethylsulfonyl fluoride $(1 \mathrm{mmol} / \mathrm{l})$, which were all purchased from Sigma-Aldrich). The protein samples were separated on polyacrylamide gels and then transferred to a nitrocellulose membrane (Bio-Rad Laboratories, Inc.). After blocking for $45 \mathrm{~min}$ in a Tris-buffered solution (TBS) containing $5 \%$ fat-free dried milk and 0.5\% Tween-20 (Sigma-Aldrich), the membrane was incubated with individual primary antibodies anti-p21, anti-p27, anti-Bcl-2, anti-Bax and anti- $\beta$-actin (1:500) overnight at $4^{\circ} \mathrm{C}$. The membrane was washed three times and incubated for $1 \mathrm{~h}$ with secondary antibodies at room temperature. The signals were visualized with the enhanced chemiluminescence method (Amersham Biosciences, Piscataway, NJ, USA). Densitometric analysis of western blots was performed using the Scion Image Beta 4.02 software (SynGene, Cambridge, UK).

Statistical analysis. All data are expressed as the mean \pm standard deviation. Statistical significance was determined using Student's t-test or one-way analysis of variance with Tukey's post hoc test. Statistical analysis was performed using SPSS 19.0 software (International Business Machines, Armonk, NY, USA). $\mathrm{P}<0.05$ was considered to indicate a statistically significant difference.

\section{Results}

SBHA impedes cell proliferation in MCF-7 cells. The CCK-8 assay revealed that SBHA treatment for $72 \mathrm{~h}$ caused a significant $(\mathrm{P}<0.05)$ inhibition of MCF-7 cell proliferation, compared to that of the untreated cells (Fig. 1). Furthermore, the inhibition occurred in a concentration-dependent manner. Since the $\mathrm{IC}_{50}$ value of SBHA in MCF-7 cells was $33.9 \pm 2.5 \mu \mathrm{M}$, an approximate concentration of the $\mathrm{IC}_{50}(40 \mu \mathrm{M})$ was used, if not stated otherwise.

SBHA induces $G_{0} / G_{1}$ cell cycle arrest in MCF-7 cells. Flow cytometry revealed that, compared with the untreated control, SBHA-treated MCF-7 cells showed a significant $(\mathrm{P}<0.05)$ increase in the $\mathrm{G}_{0} / \mathrm{G}_{1}$ phase fraction $(79.2 \pm 2.5$ vs. $38.6 \pm 2.0 \%)$ and a reduction in the $\mathrm{S}$ phase fraction $(20.4 \pm 0.8$ vs. $58.8 \pm 1.4 \%$; Fig. $2 \mathrm{~A}$ and B). Notably, SBHA appeared to induce apoptosis in MCF-7 cells, as evidenced by the appearance of a sub-G1 fraction (Fig. 2A). Western blot analysis 


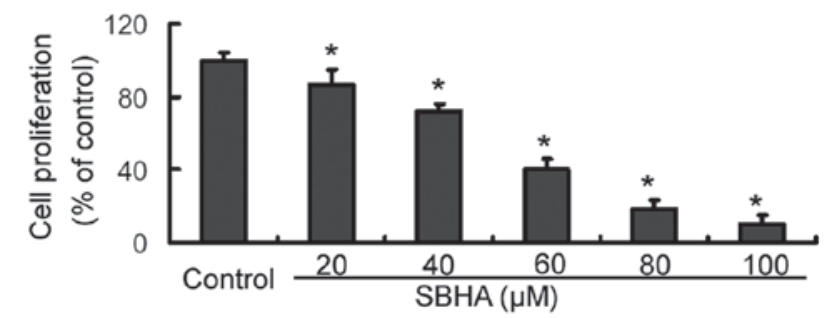

Figure 1. Suberoyl bis-hydroxamic acid (SBHA) decreases the proliferation of MCF-7 cells. Cells were treated with different concentrations of SBHA for $72 \mathrm{~h}$ and cell proliferation was assessed using the Cell Counting kit- 8 assay. The proliferation of the untreated control cells was considered to represent $100 \%$. ${ }^{*} \mathrm{P}<0.05$ vs. the control.

A

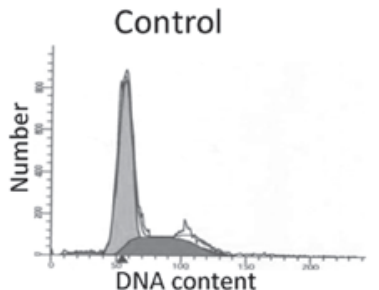

B
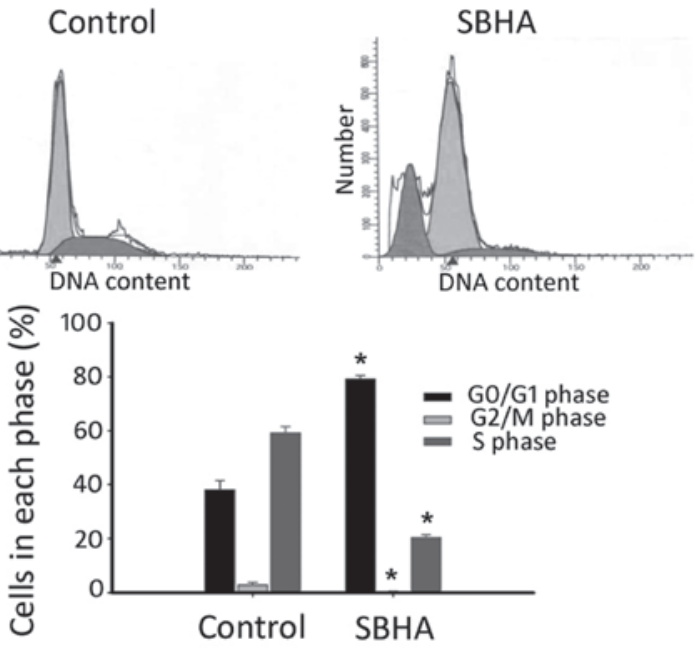

C

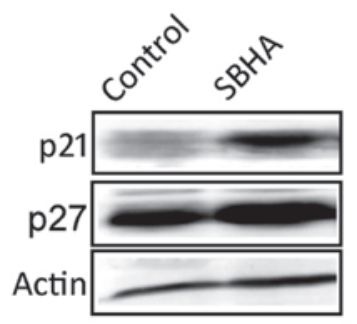

Figure 2. Suberoyl bis-hydroxamic acid (SBHA) causes cell cycle arrest at the $\mathrm{G}_{0} / \mathrm{G}_{1}$ phase in MCF-7 cells. Cells were treated with SBHA $(40 \mu \mathrm{M})$ for $72 \mathrm{~h}$ or remained untreated as a control. Cells were examined for cell cycle distribution and gene expression. (A) Representative graphs showing cell cycle distribution. (B) Bar graphs showing the percentage of cells in the $\mathrm{G}_{0} / \mathrm{G}_{1}, \mathrm{~S}$, and $\mathrm{G}_{2} / \mathrm{M}$ phases. Results are expressed as mean \pm standard deviation of three independent experiments. ${ }^{*} \mathrm{P}<0.05$ vs. the control. (C) Western blot analysis of indicated proteins. A representative blot from one of three independent experiments with similar results is shown.

demonstrated that SBHA treatment markedly raised the protein levels of p21 and p27 compared with those of the untreated cells (Fig. 2C).

SBHA promotes apoptosis in MCF-7 cells. Cell apoptosis was confirmed using Hoechst 33258 staining. The results showed that SBHA-treated cells displayed cell shrinkage, chromosomal condensation and nuclear fragmentation, which are hallmark morphological changes of apoptosis (Fig. 3A). By contrast, untreated control cells had a normal morphology (Fig. 3A). For further quantification of apoptosis, cells were stained with Annexin-V and PI and analyzed by flow cytometry. As shown
A
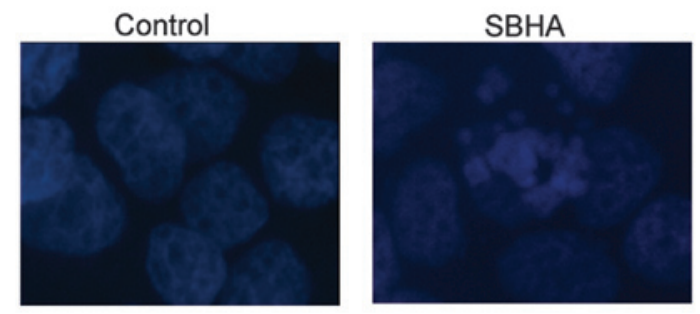

B

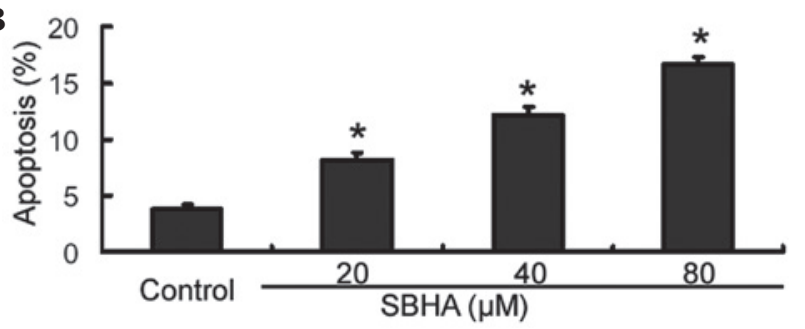

Figure 3. Suberoyl bis-hydroxamic acid (SBHA) induces apoptosis in MCF-7 cells. (A) Hoechst 33258 staining of untreated MCF-7 cells (control) and those treated with SBHA for $72 \mathrm{~h}$. (B) Apoptosis was assessed by flow cytometric analysis of Annexin V-stained cells. MCF-7 cells remained untreated (control) or were treated with different concentrations of SBHA for $72 \mathrm{~h}$ and cell apoptosis was determined. Results are expressed as the mean \pm standard deviation of three independent experiments. ${ }^{*} \mathrm{P}<0.05$ vs. the control.

A

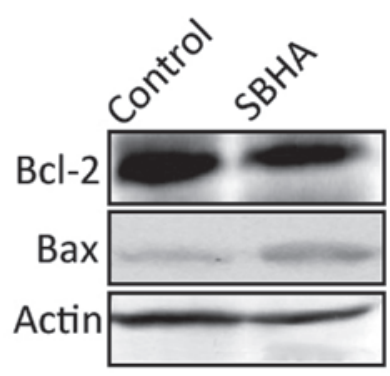

B

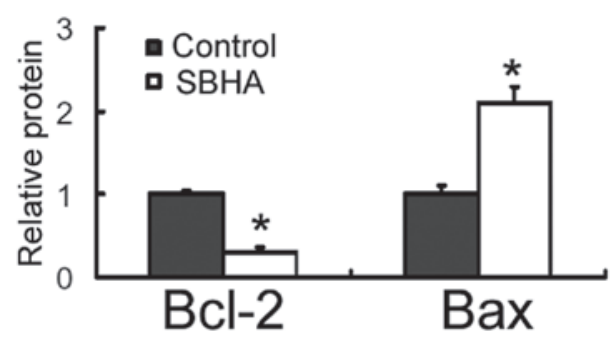

Figure 4. Suberoyl bis-hydroxamic acid (SBHA) reduces Bcl-2 expression and increases Bax expression in MCF-7 cells. (A) Representative western blot showing the modulation of Bcl-2 and Bax by SBHA. (B) Bar graph showing densitometric quantification of protein expression, represented as fold changes over control (assigned as 1). Data represent the mean \pm standard deviation of three independent experiments. ${ }^{*} \mathrm{P}<0.05$ vs. the control.

in Fig. 3B, treatment with SBHA at different concentrations for $72 \mathrm{~h}$ caused significant concentration-dependent induction of apoptosis in MCF-7 cells relative to that of the untreated control cells $(\mathrm{P}<0.05)$.

$S B H A$ reduces the $B c l-2$ expression and increases the Bax expression. Western blot analysis revealed that there was a marked reduction in the Bcl-2 protein expression level and a concomitant elevation in the Bax protein expression level in SBHA-treated MCF-7 cells, compared with those of the untreated control (Fig. 4). 


\section{Discussion}

HDAC inhibitors have been extensively investigated for their anticancer activities (13). SBHA is a relatively novel HDAC inhibitor that has demonstrated growth-suppressive effects in several types of cancer, including medullary thyroid (10) and lung cancer (11). The results of the present study showed that SBHA impeded the proliferation of MCF-7 breast cancer cells, with an $\mathrm{IC}_{50}$ value of $33.9 \pm 2.5 \mu \mathrm{M}$ after $72 \mathrm{~h}$ treatment. The antiproliferative potency of SBHA in MCF-7 cells appears to be less than that of SAHA, which has an $\mathrm{IC}_{50}$ value of $2.4 \mu \mathrm{M}$ in tamoxifen-resistant MCF-7 cells after $48 \mathrm{~h}$ treatment (9). Induction of cell cycle arrest is an important mechanism for proliferation inhibition. Notably, the current study revealed that SBHA treatment caused a significant cell cycle arrest at the $\mathrm{G}_{0} / \mathrm{G}_{1}$ phase in MCF-7 cells. Furthermore, it was determined that SBHA-treated MCF-7 cells had a marked elevation in the expression levels of p21 and p27 proteins, compared with those of the control cells. p21 and p27 are universal inhibitors of cyclin-dependent kinases and are thus involved in cell cycle control (14). Jiang et al (15) reported that genetic delivery of p21 and p27 suppresses the proliferation of MCF-7 breast cancer cells in vitro. Induction of p21 and p 27 contributes to the growth-inhibitory effect of signal transducer and activator of transcription 6 overexpression in breast cancer cells (16). These findings suggest that the antiproliferative activity of SBHA in breast cancer cells is, at least partially, mediated through upregulation of p27 and p21. SBHA-mediated cell cycle arrest has also been observed in carcinoid cancer cells (17). However, the molecular mechanisms involved in the induction of p21 and $\mathrm{p} 27$ by SBHA remain to be further defined.

Apoptosis is known as an active suicidal response that has an important role in tumor biology (18). It is characterized by cellular shrinkage without loss of plasma membrane integrity, formation of apoptotic bodies and nuclear condensation, and fragmentation. Maintenance of plasma membrane integrity during apoptosis prevents the onset of an inflammatory response that contributes to tumor progression (19). Therefore, specific induction of apoptosis represents the preferred strategy for destroying tumor cells. Notably, the results of the current study demonstrated that SBHA-treated MCF-7 cells displays apoptotic morphological changes as determined by Hoechst 33258 staining. Annexin-V/PI staining analysis further revealed that the pro-apoptotic effect of SBHA occurred in a concentration-dependent manner. These results are consistent with a previous study that demonstrated the induction of MCF-7 cell apoptosis by SBHA via a p53-dependent pathway (12).

p53-dependent induction of apoptosis is causally associated with its transcriptional regulation of numerous target genes (19). Bax is a downstream target gene of p53 that mediates p53-dependent apoptosis. It has been documented that Bax deficiency impairs p53-induced apoptosis in neurons (20). The upregulation of Bax is implicated in HDAC inhibitor-induced apoptosis in breast cancer cells (21). For instance, Wang et al (21) reported that sirtinol, a class III HDAC inhibitor, induces apoptotic death in MCF-7 cells through upregulation of Bax. SBHA has also been documented to enhance the expression of Bax in MCF-7 cells, which contributes to p53-dependent apoptosis (12). In agreement with this study, the results of the present study showed that SBHA-treated MCF-7 cells exhibited a significant increase in the Bax protein level. Furthermore, SBHA treatment significantly inhibited the expression of Bcl-2 in MCF-7 cells. Bax is a pro-apoptotic member of the Bcl-2 family. It undergoes mitochondrial intramembranous homo-oligomerization in response to apoptotic stimuli, which promotes release of cytochrome $c$ from the mitochondria, consequently activating the mitochondrial apoptotic pathway (22). The anti-apoptotic protein Bcl-2 is predominantly localized in the mitochondria and interacts with Bax to inhibit its activation (23). The results of the present study indicate that the pro-apoptotic activity of SBHA is associated with the modulation of the Bcl-2 family members. Additionally, in A549 lung cancer cells, SBHA-mediated alteration of the Bcl-2 family proteins has been reported, which leads to caspase-dependent apoptosis (11).

In conclusion, the current study revealed that SBHA exerts anticancer effects on breast cancer cells through induction of $\mathrm{G}_{0} / \mathrm{G}_{1}$ cell-cycle arrest and apoptosis. The modulation of $\mathrm{p} 21$, p27 and the Bcl-2 family proteins is involved in the cytotoxic activity of SBHA. These findings warrant further investigation of the therapeutic potential of SBHA in animal models of breast cancer.

\section{Acknowledgements}

This study was supported by the Scientific Foundation of Shanghai Municipal Health Bureau (grant no. 2012-236).

\section{References}

1. Youlden DR, Cramb SM, Dunn NA, Muller JM, Pyke CM and Baade PD: The descriptive epidemiology of female breast cancer: an international comparison of screening, incidence, survival and mortality. Cancer Epidemiol 36: 237-248, 2012.

2. Isakoff SJ: Triple-negative breast cancer: role of specific chemotherapy agents. Cancer J 16: 53-61, 2010.

3. Fukuda H, Sano N, Muto S and Horikoshi M: Simple histone acetylation plays a complex role in the regulation of gene expression. Brief Funct Genomic Proteomic 5: 190-208, 2006.

4. Federico M and Bagella L: Histone deacetylase inhibitors in the treatment of hematological malignancies and solid tumors. J Biomed Biotechnol 2011: 47564, 2011.

5. Khan $\mathrm{O}$ and La Thangue NB: HDAC inhibitors in cancer biology: emerging mechanisms and clinical applications. Immunol Cell Biol 90: 85-94, 2012.

6. Rikiishi H: Autophagic and apoptotic effects of HDAC inhibitors on cancer cells. J Biomed Biotechnol 2011: 830260, 2011.

7. Mork CN, Faller DV and Spanjaard RA: A mechanistic approach to anticancer therapy: targeting the cell cycle with histone deacetylase inhibitors. Curr Pharm Des 11: 1091-1104, 2005.

8. Alao JP, Stavropoulou AV, Lam EW and Coombes RC: Role of glycogen synthase kinase 3 beta (GSK3beta) in mediating the cytotoxic effects of the histone deacetylase inhibitor trichostatin A (TSA) in MCF-7 breast cancer cells. Mol Cancer 5: 40, 2006.

9. Lee YJ, Won AJ, Lee J, Jung JH, Yoon S, Lee BM and Kim HS: Molecular mechanism of SAHA on regulation of autophagic cell death in tamoxifen-resistant MCF-7 breast cancer cells. Int J Med Sci 9: 881-893, 2012.

10. Ning L, Jaskula-Sztul R, Kunnimalaiyaan M and Chen H: Suberoyl bishydroxamic acid activates notch1 signaling and suppresses tumor progression in an animal model of medullary thyroid carcinoma. Ann Surg Oncol 15: 2600-2605, 2008.

11. You BR and Park WH: Suberoyl bishydroxamic acid inhibits the growth of A549 lung cancer cells via caspase-dependent apoptosis. Mol Cell Biochem 344: 203-210, 2010.

12. Zhuang ZG, Fei F, Chen Y and Jin W: Suberoyl bis-hydroxamic acid induces p53-dependent apoptosis of MCF-7 breast cancer cells. Acta Pharmacol Sin 29: 1459-1466, 2008. 
13. Hrabeta J, Stiborova M, Adam V, Kizek R and Eckschlager T: Histone deacetylase inhibitors in cancer therapy. A review. Biomed Pap Med Fac Univ Palacky Olomouc Czech Repub 2013 158: 161-169, 2014.

14. Yoon MK, Mitrea DM, Ou L and Kriwacki RW: Cell cycle regulation by the intrinsically disordered proteins $\mathrm{p} 21$ and $\mathrm{p} 27$. Biochem Soc Trans 40: 981-988, 2012.

15. Jiang D, Wang X, Liu X and Li F: Gene delivery of cyclin-dependent kinase inhibitors p21 (Waf1) and p27 (Kip1) suppresses proliferation of MCF-7 breast cancer cells in vitro. Breast Cancer 21: 614-623, 2013.

16. Wei M, Liu B, Gu Q, Su L, Yu Y and Zhu Z: Stat6 cooperates with $\mathrm{Spl}$ in controlling breast cancer cell proliferation by modulating the expression of p21 (Cip1/WAF1) and p27 (Kip1). Cell Oncol (Dordr) 36: 79-93, 2013

17. Greenblatt DY, Cayo M, Ning L, et al: Suberoyl bishydroxamic acid inhibits cellular proliferation by inducing cell cycle arrest in carcinoid cancer cells. J Gastrointest Surg 11: 1515-1520, 2007.
18. Zhang L and Yu J: Role of apoptosis in colon cancer biology, therapy, and prevention. Curr Colorectal Cancer Rep 9, 2013

19. Beckta JM, Ahmad SF, Yang H and Valerie K: Revisiting p53 for cancer-specific chemo- and radiotherapy: Ten years after. Cell Cycle 13, 2014

20. Cregan SP, MacLaurin JG, Craig CG, Robertson GS, Nicholson DW, Park DS and Slack RS: Bax-dependent caspase-3 activation is a key determinant in p53-induced apoptosis in neurons. J Neurosci 19: 7860-7869, 1999.

21. Wang J, Kim TH, Ahn MY, et al: Sirtinol, a class III HDAC inhibitor, induces apoptotic and autophagic cell death in MCF-7 human breast cancer cells. Int J Oncol 41: 1101-1109, 2012.

22. Danial NN: BCL-2 family proteins: critical checkpoints of apoptotic cell death. Clin Cancer Res 13: 7254-7263, 2007.

23. Chen S, Dai Y, Pei XY and Grant S: Bim upregulation by histone deacetylase inhibitors mediates interactions with the Bcl-2 antagonist ABT-737: evidence for distinct roles for Bcl-2, Bcl-xL, and Mcl-1. Mol Cell Biol 29: 6149-6169, 2009. 